\title{
Publication of the Mathematical Works of René Thom in the Collection Documents mathématiques of the French Mathematical Society
}

\author{
Marc Chaperon (Université de Paris, France) and François Laudenbach (Université de Nantes, France)
}

Volume II has just appeared. We present the whole of the project, initiated by André Haefliger.

\section{René Thom in brief}

Thom was one of the first members of the mathematical school created around Henri Cartan at the end of the Second World War, and one of the most famous, but singular from the start: provincial, born outside of university circles, he had acquired from a very young age an intimate knowledge of differential calculus as conceived by its founders. Unusually for his time, he did not distrust geometry, where he had developed his intuition to the point of "seeing" in four dimensions. He finally followed Henri Cartan to Strasbourg as a young researcher in the CNRS, ${ }^{1}$ remaining there after the departure of his master and benefitting in particular from the influence of Charles Ehresmann.

Thus blessed with a vision of the world complementary to that of the "Parisians", Thom resolved fundamental questions which others would doubtless not even have thought of. Some landmarks:

- From 1949 to 1956, Thom worked in algebraic topology and elaborated "a completely new way of studying differentiable manifolds" (Milnor), about which he thus obtained definitive results, giving birth to the theory of cobordism, for which he was awarded the Fields medal in 1958.

- From 1956 onwards, he concentrated on the singularities of differentiable maps, which appear naturally in his vision of differential topology. Following on from Whitney, he then studied stratifications and introduced the "natural stratification of mapping spaces", defined later by John Mather. ${ }^{2}$

- From the middle of the 1960s, aware that singularities of maps and transversality aid in the understanding of all sorts of natural phenomena, he developed a catastrophe theory; this met with a period of extensive media coverage after the appearance of his book Stabilité structurelle et morphogénèse in 1972.

- At the end of the 1970s, the sometimes delirious infatuation with catastrophes came to a sudden halt, without doubt

\footnotetext{
Centre National de la Recherche Scientifique (National Center of Scientific Research)

2 This idea, to which Thom was much attached, provided for example the framework for Jean Cerf's work on pseudo-isotopy and for that of Victor Vassiliev on knot invariants.
}

hardly more justified than the vagaries of fashion. Thom then moved away from mathematics in favour of philosophy and a fertile return to Aristotle.

These changes of orientation must not mask a profound unity of thought: already on his entry to the École Normale Supérieure, Thom was tempted by the philosophy of science, from which the director of the École had to dissuade him. His great mathematical results have a strong philosophical connotation, far from the "modern" pretension of separating mathematics from the question of meaning.

\section{Birth of the project}

André Haefliger's admiration for Thom went back to their common Strasbourg years (1954-1958). ${ }^{3}$ Their relations only ceased after the disappearance of Thom in October 2002.

Already possessing documents from nearly half a century of exchange, Haefliger devoted much time from the end of 2010 onwards to the study of the Thom archives; sorted, inventoried and classified starting in mid-April 2011 in the basements of the IHES by its librarian Aurélie Brest, assisted, at the beginning, by Herminia Haefliger and himself. He discovered "veritable treasures," for example two quite amazing unpublished mathematical manuscripts, of which we will speak again. He involved Marc Chaperon in this discovery in September 2011, with the idea of setting up a "classical" paper publication, with his and Bernard Teissier's help, annotated and commented on, of the mathematical works of Thom.

Teissier and Étienne Ghys would have liked this edition to have been accompanied by the online publication of the complete works (mathematical or not) of Thom, published in the form of a CD-ROM at the beginning of 2003 by IHES. ${ }^{4}$ But alas! this quite natural idea was halted by copyright problems. ${ }^{5}$ As for the CD-ROM, perfectly usable despite the obsolescence of its search engine, it is no longer for sale.

\footnotetext{
Numerous discussions between them about foliated manifolds had then led to the "concrete" part (analytic foliations) of the still famous thesis on which Haefliger worked under the direction of Charles Ehresmann, rarely present in Strasbourg during this period.

4 Thanks to the efforts of its director, Jean-Pierre Bourguignon, and to the unfailing enthusiasm of Michèle Porte, director of this project begun in 1996 and in which Thom had actively collaborated.

5 Those for the books reproduced were ceded by their publishers only for a limited number of copies.
} 
A first editorial committee, constituted in October 2011, met at the beginning of the following month; as well as Haefliger, Teissier and Chaperon, it included Alain Chenciner. The project was submitted at the end of November by Teissier to Pierre Colmez and immediately accepted in the collection Documents mathématiques newly created by the French Mathematical Society (SMF). François Laudenbach, Jean Petitot, David Trotman and, for Volume I, Jean Lannes and Pierre Vogel quickly joined the editorial committee.

\section{Overview}

This publication does not pretend to be a substitute for the CD-ROM, but aims to complete it:

- It concentrates on the mathematical articles (or is at least classified as such in Mathematical Reviews), which are provided here with mathematical or historical commentaries, justified by later developments and the continued relevance of this often visionary work. Certain commentaries are to be found following the article which they relate to, others serve as introduction to several articles. After each article one finds shorter notes concerning precise details.

- We have chosen to reproduce the originals instead of transcribing them into modern mathematical typography, thus avoiding introducing errors and changing the page numbering. ${ }^{6}$ An exception comprises unpublished texts (absent from the CD-ROM) whose typed version was not legible enough.

As well as these unpublished texts, we are publishing a certain number of documents for their historical interest, for example large extracts from the correspondance with Cartan which led to Thom's thesis ${ }^{7}$ and fragments of letters written by him to his wife Suzanne during his stay in Princeton in 1951, which contain much information on the genesis of the work which followed.

- The bibliography, like the biographical notice, completes, corrects and enriches that of the CD-ROM, using earlier versions by Michèle Porte, Jean Petitot and Aurélie Brest, it covers all of Thom's writings, mathematical or not.

\section{Volume I}

This volume of 573 pages appeared at last in April 2017 as $\mathrm{n}^{\circ} 15$ of the collection Documents mathématiques. It covers Thom's publications from 1949 to 1959 except one, delayed until the beginning of the following volume. Given the dates, it is not surprising that it contains the major elements of Thom's work. We will evoke them a little more precisely.

The first article by Thom is a note in the Comptes Rendus de l'Académie des Sciences de Paris, 2 pages, entitled Sur une partition en cellules associée à une fonction sur une variété.

\footnotetext{
6 This choice, even if it has contributed to much delay in the publication of Volume I, was imposed all the more since Thom often published in journals or collections of articles which have become almost impossible to find.

7 We have unfortunately not been able to take into account in Volume I an inestimable document which is part of the archives donated since then by the Thom family to the library of the École Normale Supérieure: Chapter 6 on cobordism, written by Thom and excluded by Cartan, for whom it seemed to not yet make sense.
}

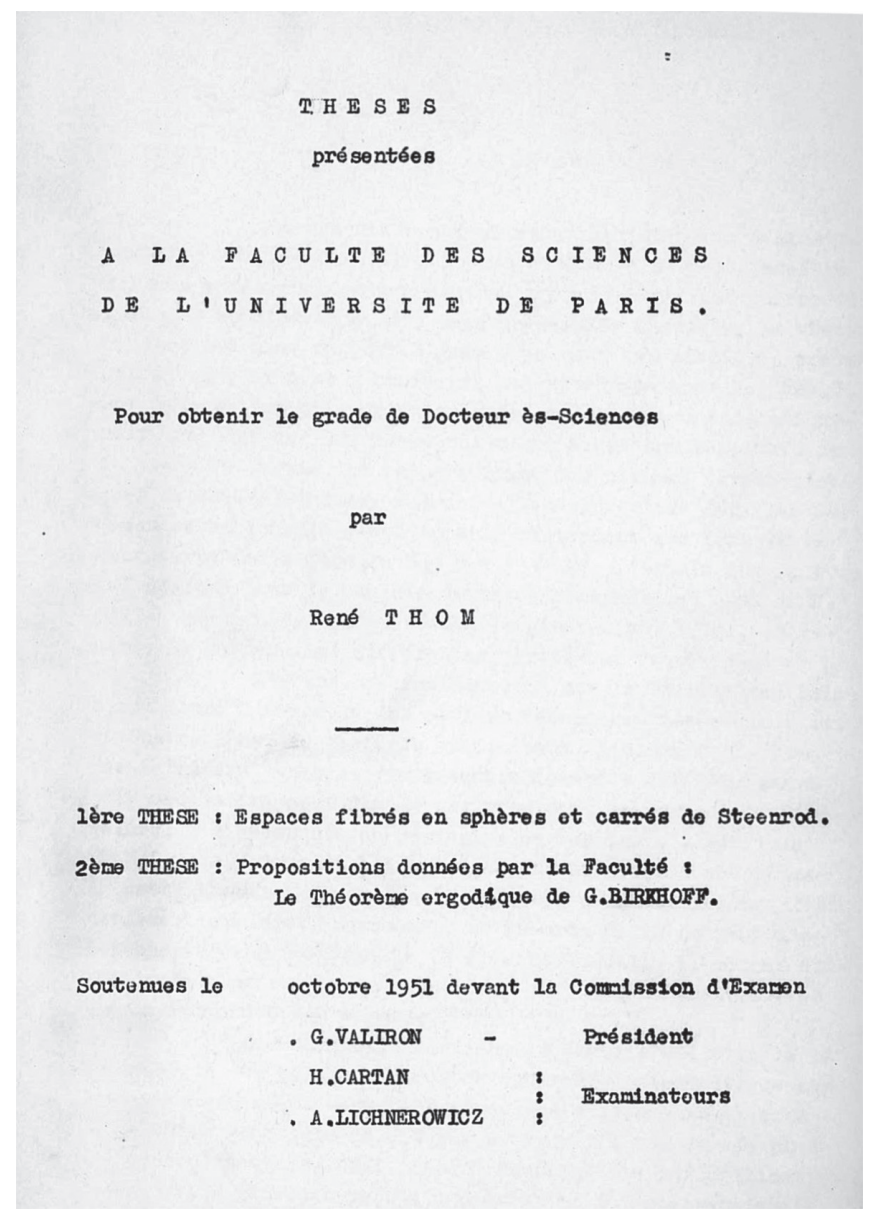

It concerns a Morse function, that is to say a function at least of class $C^{2}$ whose critical points have Hessians of maximal rank. This note has had a very rich descendance that we have made explicit by a commentary in volume I. Thom is curiously mute here about what will become, starting in 1954, one of his paradigms: the notion of genericity. There lacks indeed the supplementary hypothesis of genericity ensuring that the said partition into cells is what is called a cellular decomposition, with its specific properties concerning the attachment of the cells. It is Stephen Smale, ten years later, who will clarify this hypothesis on the gradient of the function in question, called the Morse-Smale condition today.

Next comes Thom's work on algebraic topology that we have preceded by a note of presentation written by J. Lannes and P. Vogel. This essentially covers the work by Thom for his thesis (1951) and his founding article on the theory of cobordism (1954). The thesis was published in the Annales de l'École Normale Supérieure (1952), 73 pages long.

The article from 1954, 69 pages long, has a title which looks to the future, Quelques propriétés globales des variétés différentiables. It is here that the notion of transversality appears for the first time, a property which is generically satisfied, "most of the time", by a sufficiently differentiable map. This article is important in two aspects. On the one hand it opens the way to a new branch of differential topology which will become homotopic topology. This starts from the following problem that Thom solves: under what condition on a homology class of a manifold it is realisable by a submanifold. 
On the other hand, merely by the apparition of transversality - a few pages of this article - it opens the way to the study of singularities. With some exceptions, Thom will go down this second route. Let us see what happens.

Thom will realise that the way in which he proved his transversality theorem for a submanifold applies at once, without change, to certain situations that one now calls transversality under constraint. The first of these situations is the transversality to a submanifold in a jet space.

Jet bundles were discovered by Ehresmann. For a function $f$, let us say real for simplicity, $k$ times differentiable and defined on a manifold $M$, its jet of order $k$ at a point $a$ of $M$ is, in coordinates, the Taylor development of $f$ at $a$. Of course, this polynomial depends on the coordinates, but the fact that two functions have the same Taylor development to order $k$ does not depend on coordinates. One can then collect all the $k$-jets of functions at all points of $M$ and we have thus created a new manifold $J^{k}(M, \mathbf{R})$ which fibres over $M$ by indicating the point $a$ where the $k$-jet is taken. A section of the bundle is rarely the collection of Taylor polynomials of a single function at all the points of $M$ : there is said to be an integrability condition.

Despite this constraint, the theorem of transversality to a submanifold $S$ of $J^{k}(M, \mathbf{R})$ holds, not in the space of all sections but among those which are integrable - and of course, one can replace $\mathbf{R}$ by any other manifold. However, the choice of $S$ is decisive for the study of singularities of real functions. This is what Thom does in his article, Les singularités des applications différentiables, which appeared in the Annales de l'Institut Fourier (1956). Before this paper, a "cap" written by Haefliger and based on his own archives presents the work of Thom on singularities in the period 1956-1957. Moreover, with the agreement of the American Mathematical Society, we have reproduced the long report by W. S. Massey in Math. Reviews; this sheds a useful light on Thom's article.

Because we are in 1957, let us say a few words about two unpublished papers. The first is entitled: Une démonstration d'un théorème de Lefschetz, and the second L'homologie des variétés de Stein. Thom writes at the very beginning of the second:

As a result we have the proof of a conjecture of J.-P. Serre: the homology groups $H_{i}(V, \mathbf{Z})$ of a Stein manifold of complex dimension $n$ are zero for $i>n$.

Thom presented these in a seminar in Chicago in February 1957. Why did they remain unpublished? We will never have the answer. In any case, A. Andreotti and T. Frankel published an article, The Lefschetz theorem on hyperplane sections, Annals of Math. (1959), 14 pages. In their introduction one may read:

Recently Thom has given a proof (unpublished) which, as far as we know, is the first to use Morse's theory of critical points. We present in $\$ 3$, in a slightly more general setting, an alternate proof inspired by Thom's discovery.

Let us finish this look at Volume I with a last historical evocation. Thom gave a talk in a CNRS meeting in Lille in 1959. This talk was published in the Bulletin SMF with the title Remarques sur les problèmes comportant des inéquations différentielles globales. One cannot avoid relating this title to that of the book by M. Gromov, Partial Differential Relations, Springer-Verlag, 1986; moreover, Thom's article is cited there. In this article, Thom gives, along with general considerations, a precise statement on the homology of an open set in a jet space. Here we are ten years before Gromov's thesis (1969), who proves an analogous statement but, with an important difference, Gromov speaks of homotopy. As Gromov's statement will later be called an $h$-principle after the initial letter of homotopy, one could say that Thom's statement by is an $h$-principle (only homological) before its time.

The paradox of this affair is that Thom, at the time that S. Smale wrote his (brief) report for Math. Reviews, only believed in his theorem for the jet spaces of order one as shown by Smale's commentary. However, the commentary by D. Spring in our Volume I, p. 562, specifies that he himself knew how to give a proof using the holonomic approximation theorem of Eliashberg and Mishachev (L'Enseignement Math., 2001). Finally, there is a strong chance that Thom's idea of introducing small saw teeth in his simplexes can be carried out to the end.

\section{Volume II}

As in Volume I, Thom's texts are preceded here by a complete bibliography of his works. ${ }^{8}$ It begins with the course on singularities given at Bonn in 1959, as recorded by Harold Levine. Annotated and commented by Haefliger, this lecture course is followed by a translation of the preface and the table of contents of the Russian edition by V. I. Arnold, then by a letter to Haefliger from February 1959; despite a casual error, this shows that Thom had quickly noticed the modules (moduli) which complicate the theory.

The volume then compiles the articles Thom published between 1962 and 1971, ${ }^{9}$ in chronological order of their appearance. The help of the editorial committee was solicited more here than in Volume I.

- Many of the long articles contained in the latter, written under the gaze of Henri Cartan and his school, in fact required no further commentary than the mention of their whys and wherefores. After his Fields medal, Thom is more alone but does not lose his tranquil audacity, which results in often prophetic (and, very rarely, badly written) mathematical papers requiring completion ${ }^{10}$ and, in this volume, commented on in more detail.

- This audacity led him to "step outside of the framework" with his catastrophe theory, of which the foundational articles figure in this Volume II. Sometimes containing very new mathematics, they are part of another story, notably in biology, of which it was important to give an idea: Sara Franceschelli and Jean Petitot took this on with much talent.

8 Mildly corrected with respect to Volume I, which sometimes changes the numbering.

9 Except for the Fermi Lectures Modèles mathématiques de la morphogénèse given at the Scuola Normale Superiore of Pisa in April 1971, kept for Volume III as the lectures in Bonn were kept for Volume II.

10 Mathematicians of the calibre of John Mather and Vladimir Arnold worked on this, but many exciting problems remain open. 


\section{Singularities}

A large part of this volume is formed of essential papers on singularities of maps - notably on their topological stability and on stratifications - annotated and commented upon by Teissier and Trotman, experts in the field. This includes $L a$ stabilité topologique des applications polynomiales, which already sketches the celebrated isotopy theorems of ThomMather, then Propriétés différentiables locales des ensembles analytiques (d'après $H$. Whitney), excellent exposition of fundamental results by Whitney on the stratification of analytic sets and a draft of the theory of stratified sets; this is further developed in Local topological properties of differentiable mappings, astonishingly clairvoyant, and followed by On some ideals of differentiable functions, focusing on the difference between the differentiable and the analytic.

Next there comes a "big part," Ensembles et morphismes stratifiés which, after inspiring Mather and a pleiad of other experts, has continued to be the source of active research for fifty years. The bifurcation subset of a space of maps introduces the absolutely fascinating idea of a natural stratification of function spaces, concerning which much remains to be done.

The volume concludes, in a way closer to the two unpublished papers of Volume I, with Un résultat sur la monodromie, commented on by Norbert A'Campo with Teissier, and with an unpublished manuscript on the monodromy ${ }^{11}$ which, despite a fatal error, contains important and beautiful ideas. As an anecdote, Thom's article had been accepted in a prestigious journal, which had previously simultaneously refused the paper in which the young A'Campo had given a counterexample! Happily, Thom withdrew his text and A'Campo's work, which caused the greatest surprise, established his reputation.

\section{Catastrophes}

Preceded by a knowledgeable introduction of Petitot, this part contains five articles. A dynamic theory for morphogenesis, "lost" but recovered by Tadashi Tokieda, presents the theory for the first time, insisting on its mathematical aspects - this is in particular the first appearance of universal unfoldings; the commentary led us to interrogate Mather, whose response, of great interest, is partially reproduced.

Thom presents his ideas in more detail in Une théorie dynamique de la morphogénèse, a fundamental article followed by correspondence with the great biologist C. H. Waddington; the whole piece is commented on by Sara Franceschelli and Petitot, who begin with the relation to The chemical basis of morphogenesis by Alan Turing (1952).

Then comes Topological models in biology, then A mathematical approach to morphogenesis: archetypal morphologies and Topologie et linguistique, which illustrates the scale of the project.

\section{Varia}

The volume contains other "concrete" applications of the theory of singularities: Sur la théorie des enveloppes, written in 1960 to clean up a rather "dirty" domain, is not very readable

11 In a modern typography of Duco van Straten.
- we have tried to remedy this in part without departing from Thom's ideas. Sur les variétés d'ordre fini sketches notably the proof that, save for rare exceptions, a compact submanifold $M$ of dimension $n$ in $\mathbf{R}^{n+\mu}$ cuts every affine $\mu$-plane $P$ in a finite number of points, bounded as $P$ varies.

More ambitious, the article Les symétries brisées en physique macroscopique et la mécanique quantique is confronted by Valentin Poénaru with the later progress in physics. Like the talk Travaux de Moser sur la stabilité des mouvements périodiques on "KAM theory," it bares witness to Thom's variety of interests and to his exceptional insight.

The article Sur l'homologie des variétés algébriques réelles, commented on by Ilia Itenberg, is devoted to what is often called since the Smith-Thom inequality, very important in real algebraic geometry. This work dedicated to Marston Morse depends on Morse theory, of which a "foliated" version is proposed in Généralisation de la théorie de Morse aux variétés feuilletées.

Finally Jets de Liapunov, commented on by Krzysztof Kurdyka, examines the implications of the existence of a Liapunov function for a vector field in the neighbourhood of a point.

\section{Volume III}

Respecting the name of the collection, this last volume ${ }^{12}$ essentially contains mathematical writings, a small minority of Thom's publications after 1971, but often remarkable. Let us point out some of them. The first text, the Fermi lectures Modèles mathématiques de la morphogénèse ${ }^{13}$ is a clear exposition of the stakes of catastrophe theory and a lot of new mathematics, which have had an important progeny. A critical rereading was therefore in order.

Then comes Sur le cut-locus d'une variété plongée, a paper which is very rich mathematically (it takes the functional viewpoint of The bifurcation subset of a space of maps) and even beyond mathematics - one finds there quite an up-todate model of visual perception.

Phase transitions as catastrophes which is mathematically substantial ${ }^{14}$ proposes in particular a proof of the Gibbs phase rule based on the generic structure of Maxwell sets. ${ }^{15}$ This notion was at the heart of the cut locus paper.

Sur les équations différentielles multiformes et leurs intégrales singulières provides among other things a marvellous introduction to singularities, contact geometry and Pfaffian systems.

Symmetries gained and lost analyses symmetry breakings, in connexion with the work of the physicist Louis Michel and as a continuation of the paper of Volume II.

Introduction à la dynamique qualitative, a mathematicalhistorical-philosophical text à la Thom, evidences his longstanding interest for this domain which was for a time not so popular in France, a situation which his seminar around

12 In preparation, we would like to see it published at the end of 2020.

13 It provides the first three chapters of the book with the same title published in 1974 in the pocket collection 10-18.

14 and bold, sometimes a little too much so!

15 The set of values of parameters for which a function depending on those parameters attains its minimum in several points (if one counts them with multiplicities). 
1970 had remedied, contributing to the orientation of future "leaders" in the subject, such as Michel Herman.

Gradients of analytic functions, absent from the CD-ROM and unearthed by our Iranian colleague Massoud Amini, notably states the "gradient conjecture" on the limits at a singular point of the tangents to integral curves of an analytic gradient. ${ }^{16}$ Enthusiastic commentary by Kurdyka.

Tectonique des plaques et théorie des catastrophes is the trace of a daring incursion into Claude Allègre's territory.

The Les Houches lectures Mathematical concepts in the theory of ordered media is a follow-up to the work of Kléman and Toulouse on dislocations in crystalline media.

The note with Yannick Kergosien Sur les points paraboliques des surfaces, completed and corrected with Thomas Banchoff, deals with the apparent contours of a "generic" surface in euclidean space when the direction of projection varies. ${ }^{17}$

The two notes with Peixoto on Le point de vue énumératif dans les problèmes aux limites pour les équations différentielles ordinaires, illustrate in particular the very enlightening vision that Thom had of "well posed" problems: they are those in which the $d$-dimensional manifold of solutions of the differential system is transversal in the function space to the $d$-codimensional variety defined by the limit conditions of the problem.

Quid des stratifications canoniques? evidences again their extreme importance in the eyes of Thom.

Finally, the obituaries of Morse and Whitney written for the Académie des sciences are obviously of great interest, as well as La théorie des jets et ses développements ultérieurs published with Ehresmann's complete works.

We also write a little about "the catastrophe of catastrophe theory" which took place in 1978 and without any doubt contributed to Thom distancing himself from mathematics, to its detriment.

\section{Appendix: A modest overview of transversality according to Thom}

Transversality plays an essential role in Thom's work, both in mathematics and in catastrophe theory, where a key idea is that one can only observe the phenomena that are stable under perturbation. The transversality lemma in jet spaces ${ }^{18}$ amazed its first "guinea pig" Whitney, who at first could not believe this statement which throws almost all general position arguments into the same pot. That is why we thought it would be useful to present an overview of it. ${ }^{19}$

First elementary version

The following facts seem to correspond with our intuition. If, in $\mathbf{R}^{3}$, a point $p$ lies on a surface $S$, jiggling ${ }^{20}$ will push $p$

16 A version of it has been proved by Kurdyka, Mostowski and Parusiński in a paper published in 2000 in the Annals of Math.

17 The case of a single projection was very well treated in the paper Sur les équations différentielles multiformes et leurs intégrales singulières.

18 See Thom's paper Un lemme sur les applications différentiables (1956), reproduced and commented in Volume I.

19 Marc Chaperon had treated it very differently in issue 65 of the Gazette de la $S M F$, with many other applications.

20 A generic small movement; we are borrowing this term from W. Thurston.

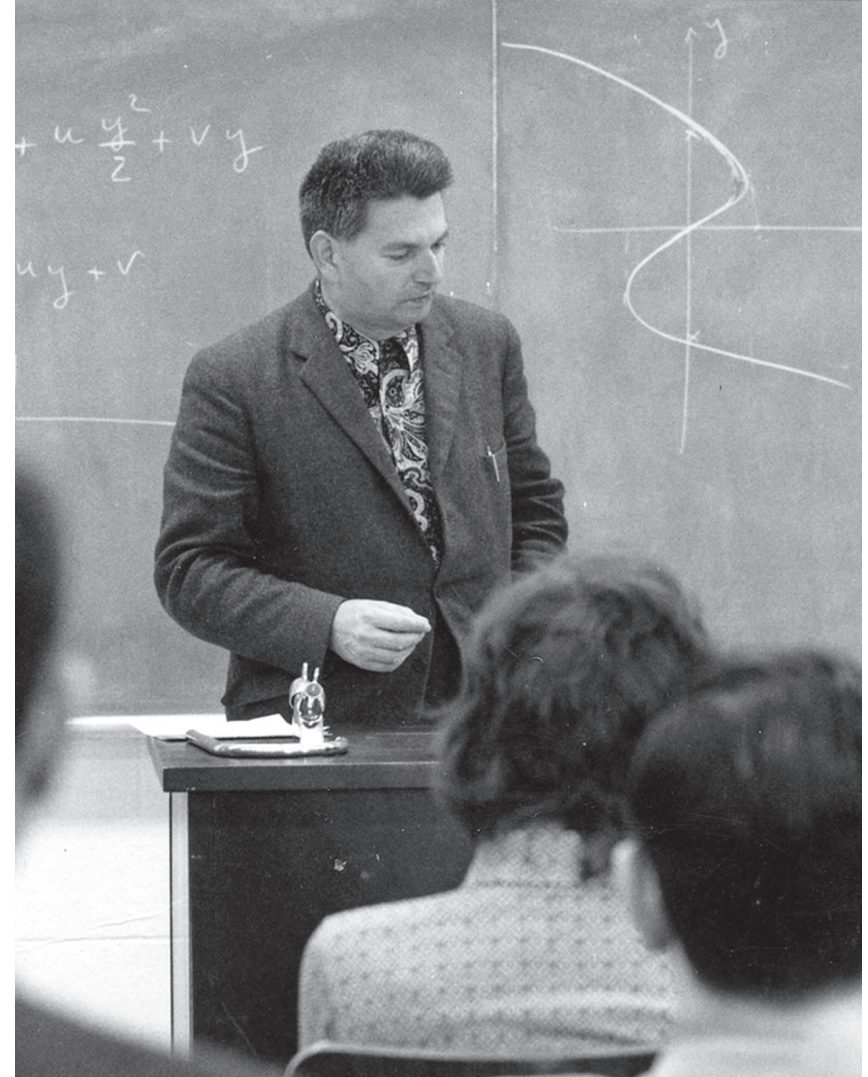

Catastrophes, around 1975 (C)Archives de l'IHÉS - Droits réservés)

outside of $S$. If a (compact) curve $C$ is tangent to $S$ at one or several of its points, jiggling will result in either $C$ and $S$ being disjoint or, at the possible common points, the tangent to $C$ and the tangent plane to $S$ will intersect transversally. Finally, if $S^{\prime}$ is a (compact) surface which at some points shares a tangent plane with $S$, jiggling of $S^{\prime}$ will put it in a transversal position with respect to $S$; at the possible common points the tangent planes will be secant; in fact - and this is less intuitive - if this last condition on the tangent planes is satisfied at each point of the intersection $S \cap S^{\prime}$, this intersection will be a smooth curve (this last fact is guaranteed by the implicit function theorem).

Thom's first statement on transversality is a generalisation on all dimensions and all manifolds of those three examples. But one has still to specify what "jiggling" is. It is a diffeomorphism $g$ of the ambient manifold $M$, arbitrarily close to the identity (in the $C^{\infty}$ topology) which one applies to a (compact) submanifold $N$ in order to make it transversal to another submanifold $N^{\prime}$ of $M$ : at any point of $g(N) \cap N^{\prime}$ the two tangent spaces $T_{x} g(N)$ and $T_{x} N^{\prime}$ generate $T_{x} M$. In what follows all manifolds will be assumed to be $C^{\infty}$ (we shall say "smooth").

Elementary transversality theorem (Thom). The property that $g(N)$ is transversal to $N^{\prime}$ is generically satisfied for $g$ in the group $\operatorname{Diff}^{k}(M)$ of $C^{k}$ diffeomorphisms of $M$ if $k>$ $\max \left(0, \operatorname{dim} N-\operatorname{codim} N^{\prime}\right)$. Here, a property is said to be generic if it is satisfied at all points of a countable intersection of dense open subsets of $\operatorname{Diff}^{k}(M)$.

Remarks. 1) If $N$ is compact, transversality is an open property in the $C^{1}$ topology and therefore in all finer topolo- 
gies such as the $C^{\infty}$ on the group $\operatorname{Diff}^{\infty}(M)$. Moreover, under this assumption, genericity is also true in the subgroup $\operatorname{Diff}_{c}^{\infty}(M)$ of diffeomeorphisms with compact support.

2) The same statement is still valid if $N$ is singular (presence of double points, lack of tangent space) or even the image in $M$ of a manifold $N$ by a $C^{k}$ map.

\section{Proof of Thom's theorem based on theorems of A. Morse} and A. Sard

Theorem (Morse-Sard). Let $X$ be a manifold. If $f: X \rightarrow \mathbf{R}^{q}$ is $C^{k}, k>\max (0, \operatorname{dim} X-q)$, then, almost every $y$ in $\mathbf{R}^{q}$, in the sense of the Lebesgue measure, is a regular value of $f$, which means that for every $x \in f^{-1}(y)$ the differential of $f$ at $x$ is of rank $q$.

For instance, if $\operatorname{dim} X<q$, almost no $y$ is in the image of $f$. By the implicit function theorem, the inverse image of a regular value is a $C^{k}$ submanifold of codimension $q$.

The inequality on $k$ in the statement above is necessary. For example, Whitney has built a function $f: \mathbf{R}^{2} \rightarrow \mathbf{R}$ of class $C^{1}$ and an $\operatorname{arc} A$ in the plane where $f$ is not constant but along which the differential of $f$ is identically zero (the $\operatorname{arc} A$ is continuous but of course not $C^{1}$ ); the interval $f(A)$ contains no regular value of $f$ although it is of positive length.

Here is how Thom uses Sard's theorem to prove his first transversality theorem. With its notations, let us consider the case $k=\infty$ and $N$ compact and concentrate on the density part. Since $\operatorname{Diff}_{c}^{\infty}(M)$ is a group one easily shows that it suffices to prove that $\mathrm{id}_{M}$ can be approximated in the $C^{\infty}$ topology by $g \in \operatorname{Diff}_{c}^{\infty}(M)$ such that $g(N)$ is transversal to $N^{\prime}$.

Thom's idea is to build, for a certain integer $r$, a map $G$ : $\mathbf{R}^{r} \times M \rightarrow M,(v, x) \mapsto G(v, x)$ of class $C^{\infty}$, such that:

(1) $G(0, x)=x$ for all $x \in M$;

(2) For all small enough fixed $v, g_{v}:=G(v,-)$ is a diffeomorphism of $M$ with compact support;

(3) $\partial_{v} G(0, x)$ is of $\operatorname{rank} n=\operatorname{dim} M$ for all $x \in N$.

We shall think of $G$ as a family $\left\{g_{v}\right\}_{v \in \mathbf{R}^{r}}$ depending on $r$ real parameters. Let us assume we have such a family and prove Thom's theorem. Since the rank condition is open, condition (3) implies that $\partial_{v} G(v, x)$ is of rank $n$ for all $(v, x)$ in a neighbourhood $U$ of $\{0\} \times N$. Let us still denote by $G$ its restriction to $U$. With the rank condition, the implicit function theorem tells us that $W:=G^{-1}\left(N^{\prime}\right)$ is a submanifold of $U$ of class $C^{\infty}$.

Let $\Pi: W \rightarrow \mathbf{R}^{r}$ be the projection onto the parameter space. One now applies Sard's theorem to П. Almost every ( $r$-dimensional) parameter $v$ is a regular value of $\Pi$. An easy lemma of linear algebra shows that for such a $v$, the diffeomorphism $g_{v}$ pushes $N$ to a position transversal to $N^{\prime}$.

It remains only to build the family $G$. Let us begin with the case $M=\mathbf{R}^{n}$. We take as parameter space the vector space $\overrightarrow{\mathbf{R}}^{n}$ of translations of $\mathbf{R}^{n}$. By a well chosen partition of unity, one extends the translation of the compact $N$ by the vector $v$ into a diffeomorphism $g_{v}$ with compact support which is $\operatorname{id}_{M}$ if $v=0$. Conditions (1) $-(3)$ are satisfied.

In the case where $M$ is a manifold, using the compactness of $N$ we cover it by a finite collection $\left\{B_{i}\right\}_{i=1}^{s}$ of balls, each of which is contained in a coordinate chart $O_{i}$ of $M$.

One chooses the translations $v_{i}$ in the coordinates of $O_{i}$ small enough for $B_{i}+v_{i}$ to remain in a fixed compact subset of this chart. One easily extends this translation into a dif- feomorphism $g_{i, v_{i}}$ of $M$ with compact support which is $\operatorname{id}_{M}$ if $v_{i}=0$. Let $v:=\left(v_{1}, \ldots, v_{s}\right)$. Set $g_{v}=g_{1, v_{1}} \circ \cdots \circ g_{s, v_{s}}$. One easily verifies that conditions (1)-(3) are satisfied, which ends the proof.

\section{Transversality and jet spaces}

It is obviously in the jet spaces of order $r \geq 2$ that Thom will give the deepest applications of the transversality theorem to singularities of differentiable mappings. However, one can already realise the innovative aspect of this theorem with jets of order 1 (or 1-jets) of real functions of one real variable.

In order to start with a compact manifold, let us look at the 1 -jets of functions on the interval $[0,1]$, which is not exactly a manifold, but a manifold with boundary. The boundary creates no difficulty for understanding the space of real valued $C^{\infty}$ functions.

If $f:[0,1] \rightarrow \mathbf{R}$ is $C^{k}, k \geq 1$, we define its jet of order one $j^{1} f(a)=\left(a, f(a), f^{\prime}(a)\right)$ at any point $a \in[0,1] .{ }^{21}$ The map $j^{1} f$ is a section (that is, a right inverse) of the projection $(x, y, z) \mapsto x$ of $J^{1}([0,1])$ to $[0,1]$; thus, it identifies with its image which is a $C^{k-1}$ submanifold of $J^{1}([0,1])$.

Given $a \in[0,1]$, any $P \in \mathbf{R}[T]$ of degree one is equal to $j_{a}^{1} f$, where $f(x)=P(x-a)$. However a global section $s(x)=(x, y(x), z(x))$ is of the form $j^{1} f(x)$ if and only if

$$
z(x)=\frac{d y}{d x}(x) \quad \text { (integrability condition). }
$$

This condition is quite rarely satisfied. Thus, if $\Sigma$ is a submanifold of $J^{1}([0,1])$, the proof given for the transversality theorem (elementary version) will produce from $j^{1} f$ at best a section transversal to $\Sigma$, with practically no chance to produce an integrable section.

We remember that everything lies in the choice of the family $G$ of maximal rank with respect to the parameters. It is Thom's clairvoyance which made him see that the same proof worked in the subspace of integrable sections if we take as parameter space the (finite dimensional) space of degree one real polynomials, here in one variable. At the same time we shall replace the diffeomorphism group of $J^{1}([0,1])$ by its subgroup, which one might call the gauge group consisting of those diffeomorphisms which preserve not only each fiber $J_{x}^{1}([0,1])$ but also the distribution of contact planes, i.e., the kernels of the differential form $d y-z d x .^{22}$

If $P$ is a polynomial function of degree one, the translation (in the fibers)

$$
\begin{aligned}
& (x, y, z) \mapsto\left(x, y+P(x), z+P^{\prime}(x)\right) \\
& \quad \text { in other words } \quad j^{1} f(x) \mapsto j^{1}(f+P)(x)
\end{aligned}
$$

is a gauge transformation, sending each integrable section $j^{1} f$ to the integrable section $j^{1}(f+P)$. This family of translations verifies conditions (1)-(3), once truncated to obtain (2).

The same approach works in any dimension of base manifold and for any jet order. ${ }^{23}$ Thom thus obtains the following theorem:

\footnotetext{
21 In other words $j^{1} f(a)=\left(a, j_{a}^{1} f\right)$, where $j_{a}^{1} f \in \mathbf{R}[T]$ is the Taylor polynomial $f(a)+f^{\prime}(a) T$

22 A "contactologist" tradition exchanges $y$ and $z$, giving $d z-y d x$.

23 Even replacing the target space $\mathbf{R}$ by another manifold.
} 
Transversality theorem in a jet space (Thom). Let $N$ be an $n$-dimensional manifold, let $\mathrm{J}^{r}(N)$ be its space of $r$-jets of real functions and let $\Sigma$ be a submanifold of $J^{r}(N)$ of codimension $q$ and of class $C^{\ell}, \ell>\max \{0, n-q\}$. For any $f \in C^{k}(N, \mathbf{R})$, $k-r>\max \{0, n-q\}$, generically for $g$ in the $C^{k-r}$ gauge group, the submanifold $g\left(j^{r} f(N)\right)$ is transversal to $\Sigma$.

Here is a first application to Morse functions. At the beginning of the 1930s, Marston Morse showed the importance of the functions which now bear his name for the understanding of manifolds. Recall that a Morse function $f: N \rightarrow \mathbf{R}$ is a function, of class at least $C^{2}$, whose critical points (points where $d f=0$ ) are all non degenerate (in coordinates, the matrix of second partial derivatives is of maximum rank).

Corollary. Morse functions of class $C^{k}$ on $N$ are dense in $C^{k}(N, \mathbf{R})$ for $k \geq 2$.

Indeed, a simple computation shows that $f$ is a Morse function if and only if $j^{1} f$ is transversal to the zero section $\Sigma:=\left\{j^{1} \varphi(x): d_{x} \varphi=0\right\}$ of the projection $j^{1} \varphi(x) \mapsto j^{0} \varphi(x):=$ $(x, \varphi(x))$. According to the theorem (here with $q=n$ and $r=1$ ), generically $j^{1} f$ is transversal to $\Sigma$, hence the density.

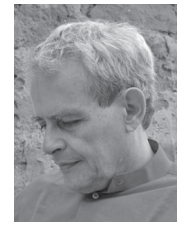

Marc Chaperon [marc.chaperon@imj-prg.fr], currently a professor emeritus at the university of Paris, is a former student of the École normale supérieure. As a beginner, he received the advice of René Thom, Alain Chenciner and Michael R. Herman. His work in differential geometry deals with dynamical systems, singularities and symplectic geometry.

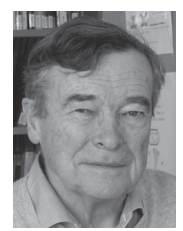

François Laudenbach [francois.laudenbach@ univ-nantes.fr] is a topologist (Pseudo-isotopy, 2-spheres in 3-manifolds, generating functions in symplectic topology, Morse-Novikov theory). He was awarded Peccot lectures (Collège de France, 1973). He had professor positions successively in Université Paris-sud (Orsay), École Normale Supérieure de Lyon, École polytechnique, Université de Nantes where he retired as Professor emeritus. 